NOTA

265-273

\title{
LA DESARTICULACIÓN DE LO HABITUAL. CONSIDERACIONES FENOMENOLÓGICAS SOBRE EL ESPACIO A PARTIR DE LA INTERVENCIÓN ARQUITECTÓNICA HAUS U R DE GREGOR SCHNEIDER
}

The disarticulation of the habitual. Fenomenological considerations on space according to the arquitectonic intervention Haus u r of Gregor Schnider

Pía Cordero*

\section{INTRODUCCIÓN}

A partir de la segunda mitad del siglo XX la escultura se desplaza a la conquista del lugar, desde el minimalismo se transgrede el canon escultórico para acceder al territorio; se abandona el concepto de monumentalismo para problematizar el lugar de la obra; surge la noción site specific, cuya transversalidad abarca disciplinas tales como artes visuales y arquitectura, así también teatro, danza y performance. Consecuentemente, debido a la integración de elementos somáticos se reorienta la percepción, para tomar distancia de una visión del arte dominada por la perspectiva visual. En el contexto de la transgresión y deconstrucción artística de los últimos decenios - en cuyos hitos destacan, por ejemplo, la Merzbau de Kurt Schwitters (1887-1948) y la anarquitectura de Gordon Matta-Clark (1943-1978)-, se hace necesario repensar aquello llamado espacio, con el fin de comprender las operaciones del arte, cuyos desplazamientos se originan en aquello común y unitario llamado mundo. En estas coordenadas, destaca el trabajo Haus $u$ r, del artista alemán Gregor Schneider, intervención arquitectónica realizada durante 16 años (19852001). En 1985 el artista comenzó a intervenir la casa donde habitaba, duplicando paredes y ventanas, creando nuevos lugares con la sala de estar, el dormitorio, la cocina, el pasillo, el cuarto de invitados y el sótano. En efecto, realiza habitaciones concéntricas, laberintos y accesos escondidos, bajo una poética arquitectónica del vacío y la desestabilización. En 1988 realiza la intervención $u r 1 u$ 15, habitación dentro de otra habitación, construida con placas de yeso y madera, iluminada con lámparas halógenas y un ventilador, que ubicados en el intersticio de las habitaciones simulan luz y brisa matutina. En u r 8 (19891991), habitación aislada e insonorizada con puntas de espuma, construida con plomo, lana de vidrio y materiales para la aislación sonora, la oscuridad y el aislamiento de los tránsitos constructivos de la obra, llevan el habitar a la indeterminación y hermetismo. En tanto Haus $u$ r refiere al habitar dentro de una casa y su emplazamiento en la ciudad ¿A qué vivencia de campos sensibles está referida? ¿Se aleja de los cánones visuales ideales y abstractos del museo y de la exposición? En La obra de arte en la época de su reproductibilidad técnica, Walter Benjamin examina los aspectos táctiles de la obra de arte presentes en la aprehensión sensible de las construcciones mediante el desplazamiento, el uso y la proximidad. Asimismo, en el año 1990, en su ensayo Llamado 
al orden. En defensa de la tectónica, Kenneth Frampton refiere a lo tectónico como estrategia estética para la aprehensión de la obra construida y su entorno, alternativa crítica a la escenografía y simulacro característicos de la arquitectura posmoderna. Según Frampton, los elementos táctiles efectúan:

El deseo transvanguardista de retornar a la atemporalidad del pasado prehistórico, para recuperar esta dimensión de un presente eterno por fuera de las pesadillas de la historia y las compulsiones del progreso instrumental. (...) Dentro de la arquitectura, la tectónica aparece como una categoría mítica a través de la cual ingresar a un mundo donde la "presencia" de las cosas facilite la aparición y experiencia de los hombres (3).

Siguiendo a Benjamin y a Frampton es posible afirmar que desplazamiento, uso, proximidad, forma construida y estructura, constituyen partes de la estrategia táctil, vivencia de la corporalidad del espectador, que constituye en una intervención como Haus $u r$, el medio inmediato y casi indescifrable en el que acontece la obra: movimiento, fricción y adherencia. Además, en el contexto de la relacionalidad, cuyo eje fundamental es la interacción y la afirmación de la ciudad en su experimentación espacio-temporal, la obra de arte es apertura y disolución de sus límites en la urbe, es proximidad, es decir, “(...) un estado de encuentro (...)" (Bourriaud, 15). En esta perspectiva, resulta importante mencionar la diferencia efectuada por Bourriaud entre forma y formaciones: en oposición al hermetismo tradicional de la forma, las formaciones mientan los movimientos de encuentro y de intercambio de experiencias. Por este motivo, caracteriza el trabajo artístico la intersubjetividad, medio por el cual aquello que comparece se torna próximo. El estado intersubjetivo que supone la práctica relacional del arte no se origina en la interioridad subjetiva ni en la exterioridad del objeto, sino que emerge del mundo como acontecer de lo que separa y a la vez reúne. En este sentido, cabe destacar lo que Jacques Derrida plantea sobre el lugar (khorra), que contrario a todo tipo de polaridad nominativa -sujetoobjeto, negativo-positivo, si-no es un triton genos: "No es el sujeto. Ni el subjetil (subjectile). Los tipos hermenéuticos no pueden informar a khôra, no pueden darle forma, como no sea en la medida en que, inaccesible, impasible, "amorfa" y siempre virgen, de una virginidad radicalmente rebelde al antropomorfismo, parece recibir esos tipos y darles lugar" (27). Al igual que un triton genos el arte relacional otorga parte a aquello innombrable, no como imposibilidad, sino como ruptura con el tradicional sentido que ha dominado gran parte de las categorizaciones y nominaciones estéticas. De esta manera, la práctica artística expande sus límites para emerger del mundo como hito y acontecimiento reclamando un lugar para sí. En efecto, Haus $u r$ al devenir entre lo doméstico y lo estético, emerge como replanteamiento crítico de la relación obra-hombre. En esta tensión la obra como espacio privado, subyugado a satisfacer los aspectos más simples del diario vivir, y como espacio estético, cuando las habitaciones refieren a algo más que a la simple utilidad, evidencia la necesidad de problematizar qué es el espacio, que deviene forma construida y que en su inaccesibilidad tuerce el esquema lógico de la arquitectura y funcionalidad de una casa. En ésta, las cualidades más sutiles del habitar como el caminar 
seguro y el conocimiento de la disposición de los lugares convergen en el desconocimiento de lo que está a la mano, dislocando la percepción en la ocupación y el uso. La tarea de reflexionar sobre el espacio contemporáneo en su hibridación y transgresión estética lleva a pensar sobre el concepto de límite, en tanto el espacio es concebido como tránsito, no-lugar, fragmentación, espuma, etcétera, es decir, posibilidad de quebrantar su sentido. No obstante, pensar el espacio en lo múltiple de sus manifestaciones es permanecer en lo estático de un conocimiento, que desde la memoria del topos a la no-memoria de la indeterminación posmoderna, contribuye a hacer asible la realidad concebida como organización o caos. Sin embargo, ¿Cómo tomar distancia de una interpretación sustancial o indeterminada del espacio? o como plantea Gaston Bachelard, quien afirma: “(...) la expresión crea ser. (...) pensamos que todo lo que es específicamente humano en el hombre es logos. No alcanzamos a meditar en una región que existiría antes que el lenguaje" (15), ¿Constituye una imposibilidad meditar sobre el espacio de modo anterior al logos? Heidegger reflexiona sobre el espacio como estructura existencial referida al "ser-ahí" del hombre. El estudio de la relación del hombre con aquello que cotidianamente le circunda, surge a partir de su consideración como un ser situado, que a partir del ahí $(\mathrm{Da})$ de su ser es capaz de proyectarse lejos de una determinación topográfica como límite y mera ubicación de algo. Esto es posible porque el hombre descubre lo que está ahí delante, ya sea mediante su empleo o imposibilidad de uso, consecuencia de su estructuctura existencial "estar-en-el-mundo". Afirma Heidegger que antes de toda categorización y determinación del hombre está aquello que "le va en su ser este mismo ser" (Ser y tiempo, 35), esto es, el Dasein, aquel ente que se auto comprende en su propia determinación. En estos lineamientos se articula una reflexión sobre el espacio que emerge del mundo habitual del hombre y que mediante la estetización de una vivienda, que problematiza sus límites desde el cuestionamiento al confort y la autosuficiencia doméstica, hace emerger la siguiente interrogante $¿ E s$ acaso el espacio estético contemporáneo un deshabitar, es decir, una huida ante las relaciones cotidianas del hombre?

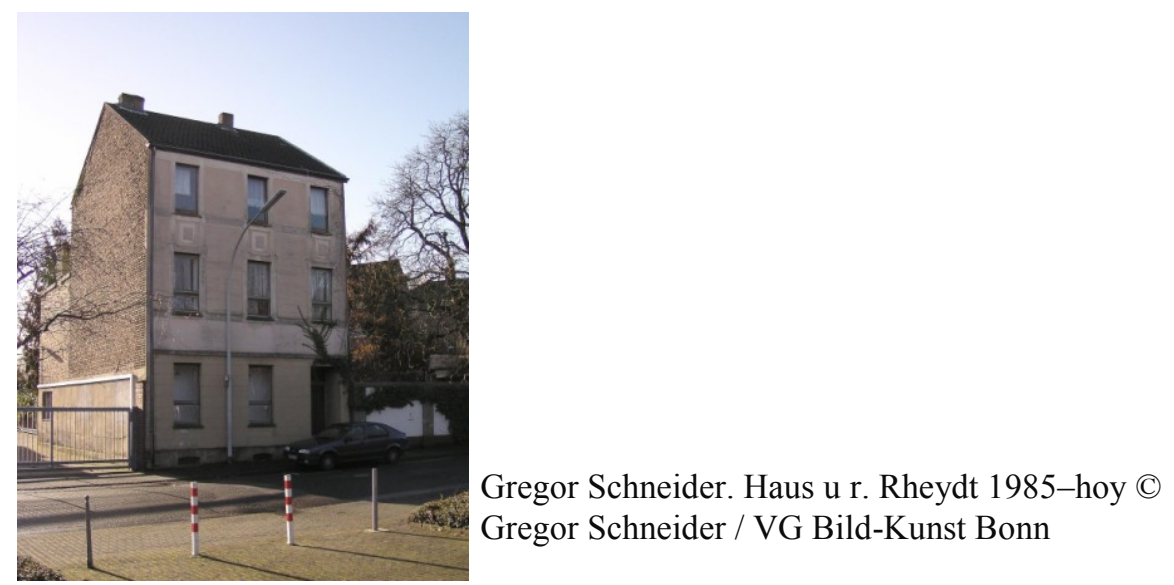




\section{LA ARTICULACIÓN DE LO HABITUAL}

En Ser y tiempo Heidegger analiza el útil (Zeug) o ser a la mano (Zuhandenheit) presente en la vida del hombre de manera significativa. En lo cotidiano (Umwelt) los útiles que conforman el mundo aparecen bajo la figura de la empleablidad e inempleabilidad, esto es, bajo la posibilidad o imposibilidad de uso. En la ocupación el útil emerge como un "para-algo", no comportando ningún tipo de tematización u objetivación teórica, como afirma Heidegger: "Un útil no es en rigor jamás. El útil es "algo para..." (Ser y tiempo 96). De hecho, generalmente, una escalera, una mesa o una silla conforman el mundo en un silente "para-algo", estando disponibles para su ocupación, en oposición al observar distante de la teorización y contemplación estética. Asimismo, en El origen de la obra de arte el filósofo examina el útil desde la noción de instrumento determinado por el uso habitual y confiable. Las cualidades del ser útil son resumidas en dos: se gasta o consume, y se vuelve habitual. Cuando la utilidad desaparece por causa del desgaste se suprime su rasgo esencial: su ser de confianza, en donde descansa su apariencia estable. En efecto, una casa y sus enseres se caracterizan por el uso y la habitualidad de lo familiar, y su ser de confianza yace en la seguridad y protección de su resguardo. Por el contrario, en la desarticulación de lo habitual, una intervención artística como Haus u $r$ desplaza la servicialidad y el ser de confianza del útil al más simple estar-ahí al descubierto. Es el caso de la intervención ur 9 u 42 (1992), cielo raso móvil debajo de techo, construido con placas de aglomerado de fibras de madera, sobre construcción de vigas con motor. En este trabajo Schneider interviene el techo de una de las habitaciones de su vivienda con el fin de mover el cielo raso, el cual imperceptiblemente sube y baja mediante la fuerza de un motor. También destaca la intervención ur 10 (1993), construida con partes de ur 3 (1986), habitación giratoria dentro de habitación -la distancia que separa a las habitaciones concéntricas es aproximadamente de 35 a $105 \mathrm{~cm}$ - construida con placas de yeso y de madera aglomerada, sobre construcción de vigas con postes, rodillos y motor. En este caso, la habitación interior gira levemente desorientando la percepción del espacio.

El hombre se encuentra en constante relación con las cosas del mundo, sin embargo, para que éstas comparezcan en su particularidad deben tener un contexto previo que las determine. Por ejemplo, la significación de una mesa para cenar o cocinar está determinada por la totalidad en la que se encuentra: sillas, enseres de cocina, alimentos, cubiertos, etcétera, mientras que la significación de la misma mesa para diseccionar un cadáver está dada por los instrumentos: bata, gorro quirúrgico, bisturí, pinzas, ganchos, sutura, sondas, etc. En otras palabras, el desplazamiento del "estar-ahí" del útil al "paraalgo" de su ocupación es efectuado desde la referencia a las cosas en las que se encuentra, que en su totalidad generan la figura del mundo. Al respecto dice Heidegger:

Este ente a la mano, que por eso llamamos martillo, está en respectividad con el martillar, el martillar lo está con el clavar y consolidar, éste lo está con la protección contra el mal tiempo; y esta última "es" por mor del Dasein (3) que necesita protección, es decir, por mor de una posibilidad de su ser (Ser y tiempo, 110). 
El útil se caracteriza por su lugar propio (Platz). Cualidad que refiere a su disponibilidad en la totalidad de pertenencia, y que no implica estar ocupando un sitio mediante los límites que supone la extensión de un cuerpo. En este sentido, el espacio habitual se articula a partir del trato del hombre con las cosas del mundo, las cuales tienen dos modos de espacialidad: en base al lugar propio en el estar a la mano -que corresponde a un aquí o ahí- o según el adónde (Wohin) que constituye una zona (Gegend), totalidad del conjunto de útiles. En ambos casos, el hombre existente se relaciona con las cosas bajo la des-alejación (Ent-fernung) y la direccionalidad (Ausrichtung), que explican la capacidad del Dasein de hacer desaparecer las distancias, mediante el: "procurarse (algo), aprestarlo y tenerlo a la mano (Heidegger, Ser y tiempo, 131). Esta puesta en libertad permite que lo espacial del mundo circundante pueda comparecer como apertura del espacio. Esto es, la comparecencia del ente en el mundo conlleva un "abrir espacio" (Raumgeben) o una "ordenación espaciante" (Einräumen), posibilidad de que el ente a la mano pueda ser dejado en libertad, promoviendo su posterior orientación en el quehacer habitual. Así entendido, el espacio no equivale a una objetivación, que establece determinaciones de grado entre lo que es y no es espacial, al contrario, la espacialidad emerge en lo absorto de la ocupación cotidiana. Consecuentemente, mediante la noción de cercanía se articula la caracterización de espacio y existencia como nociones correspondientes, en tanto el Dasein no es estático, pues se determina desde la referencia a las cosas en las que está inmerso, dando lugar a su aquí mediante el trato habitual con los entes del mundo. Al respecto afirma Heidegger: "El Dasein comprende su aquí desde el allí del mundo circundante. El aquí no mienta el dónde de algo que estuviera-ahí, sino el en-medio-de-qué de un desalejante estar en-medio-de (...) y junto con él la desalejación misma" (Ser y tiempo, 133).

De lo enunciado en los párrafos anteriores cabe preguntar ¿Cuál es el lugar del cuerpo que hace frente en la vida cotidiana y encarna la existencia? el Dasein como despejamiento, éxtasis y acaecimiento de sí, viviendo el cuerpo que es, esgrime sus distancias y cercanías en relación a las cosas que le rodean. Es decir, la relación del Dasein con el cuerpo no refiere a una dimensión física, sino a la vivencia de su corporalidad, emplazada en el entramado de la cotidianeidad, estructura impropia que determina la facticidad de la existencia. Esta vivencia, según Heidegger, se caracteriza por un "aguantar espacios" (Construir, 116), posibilidad de desplazamientos que atraviesa y dispone el residir en los lugares. Finalmente, es posible afirmar que la articulación de lo habitual reside en la existencia que estructura, mediante la caída del Dasein, el mundo fáctico. Permanente impropiedad (Uneigentlichkeit), en la cual no es dueño de sí mismo, sino, por el contrario, está sometido al arbitrio de lo uno (Man), en donde comparecen los otros en sus ocupaciones y modos de ser. Desde esta perspectiva, acontece el residir del hombre en el trato habitual con lo que tiene a la mano, desde una dimensión originaria y no tematizada, que posibilita la referencia a las cosas del mundo y la tarea, llámese filosófica, de "llegar a encontrarse". 


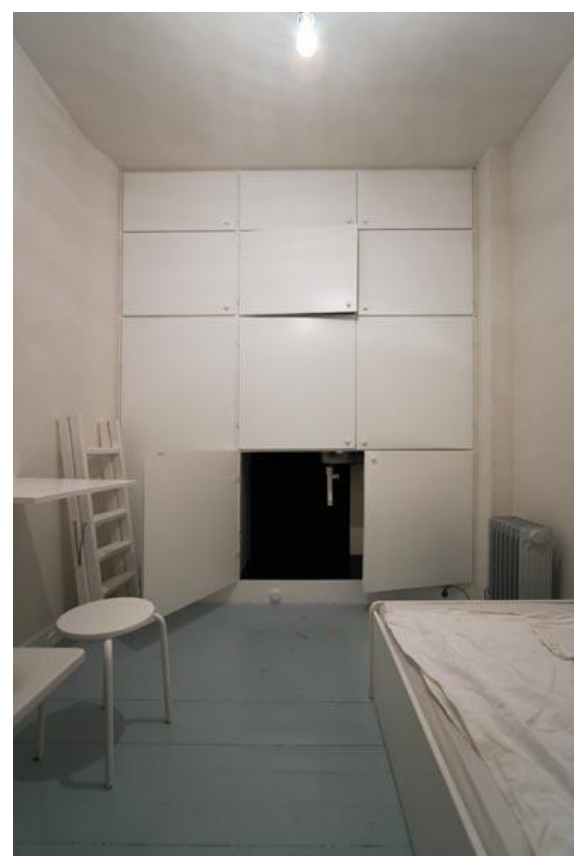

Gregor Schneider. U r 19,

LIEBESLAUBE, Haus u r. Rheydt 1995-96 C Gregor

Gregor Schneider. U r 19, LIEBESLAUBE, Haus u r. Rheydt 1995-96 C Gregor 


\section{LA DESARTICULACIÓN DE LO HABITUAL}

Lo cotidiano se caracteriza por estar sumido ante las ocupaciones, caída en la impropiedad del mundo que configura la vida fáctica. En este contexto, Gregor Schneider en su obra Haus $u r$ modula un argumento de creación imbricado que destruye la unidad funcional de una construcción habitacional mediante la duplicación de accesos y habitaciones. Las inacabadas modificaciones que recibe la casa surgen como intervenciones artísticas, cuyos códigos arquitectónicos dejan la estabilidad del lenguaje, para constituirse en indeterminación de los límites del espacio habitable. Más aún, mediante la pugna y confrontación con lo habitual recrea la relación hombre y espacio, a través de la resignificación de los dinamismos que determinan las referencias a las cosas. Desde esta perspectiva, ¿Cómo se caracteriza aquello que disloca el sentido de lo habitual?¿Cómo una intervención artística puede dejar entre paréntesis las significaciones del diario vivir?

Según Gianni Vattimo la sociedad de las comunicaciones promueve el surgimiento de las heterotopías en oposición a una visión unitaria de la historia. Sobre los mass media y su influencia en la idea de la historia como fragmentación afirma: “(...) a pesar de cualquier esfuerzo por parte de los monopolios y las grandes centrales capitalistas, es, más bien al contrario, que la radio, la televisión y los periódicos se han convertido en componentes de una explosión y multiplicación generalizada de Weltanschauungen: de visiones del mundo" (79). Sin embargo, afirma el filósofo, en la extrañeza del hombre frente a las diferencias, es decir, bajo el supuesto de la fragmentación y el desconocimiento, los medios de comunicación generan una suerte de autoconciencia de la simultaneidad. Consecuentemente, el hombre es consciente de sus límites como parte de una totalidad, suerte de extrañamiento ante la emergencia de los elementos locales, los dialectos y las diversidades. En este contexto, la obra de arte es caracterizada por Vattimo como "oscilación entre la pertenencia y el extrañamiento", (152), interpretación efectuada a partir de los conceptos Schok de Benjamin y Stoss de Heidegger, cuya función según Vattimo es: "captar los rasgos principales de la nueva "esencia" del arte en la sociedad tardoindustrial; rasgos en que la reflexión estética contemporánea, incluso la más penetrante y radical -empezando por Adorno- no ha reparado" (137). Por una parte, Benjamin observa en la obra de arte un desplazamiento desde lo aurático, caracterizado por la unicidad y perdurabilidad, a lo profano, representado por lo reproducible y exhibible. Tránsito provocado por el desarrollo de los medios de reproducción técnica y el complejo entramado de aparatos tecnológicos que modelan paulatinamente el acontecer del hombre. En efecto, para Benjamin, el cine produce aquel Shock cuando quienes observan la imagen cinematográfica perciben con mayor fuerza el mundo mediante la reproducción técnica de: imagen, sonidos, escenografia, interpretaciones, etc. De modo semejante, el Stoss heideggeriano es angustia ante el desarraigo, que yace en la confrontación del ser obra como apertura y develación de la verdad. Afirma Heidegger, que cuando se contempla la obra surge un "extático abandonarse del hombre existente a la desocultación del ser" (El origen, 79), el cual desarticula el entramado de las relaciones 
cotidianas con las cosas del mundo. Para el filósofo, la obra de arte es conflicto en la exposición del mundo y la producción de la tierra. Particularmente, la tierra que confronta y de la cual emerge posee el carácter de la inagotabilidad, es decir, es $\varphi u ́ \sigma \iota \varsigma$, " (...) lo que brota y surge por su propia fuerza y sin apremio alguno, lo que vuelve sobre sí y perece: el imperar que brota y vuelve a sí" (Heidegger, Nietzsche, 85), y el mundo que expone es el sistema de significados que inaugura, los que extrañan causando: "la movilidad y la hipersensibilidad de los nervios y de la inteligencia, características del hombre urbano" (Vattimo, 151). Cuando se contempla la obra surge según Heidegger: "un extático abandonarse del hombre existente a la desocultación del ser" (El origen, 79), que desarticula las relaciones de las cosas del mundo. Es preciso señalar que devenir y acontecimiento de la obra de arte constituyen una lucha, tensión entre "alumbramiento" y "ocultación", que manifiesta el modo de su estar al descubierto. Retomando el análisis de Haus $u r$, la apertura de la obra se origina desde las intervenciones artísticas a una casa y de quien las vivifica bajo la desorientación y el asombro. Relación controversial en tanto el acontecer de la obra efectúa su estetización desde la escisión de la casa-útil a la casa-obra o desde el para-sí al en-sí de su estar. Bajo la interpretación del Stoss heideggeriano como sensación de extrañamiento, una intervención artística a una casa se torna desazón, es decir, angustia ante la carencia de la seguridad de lo familiar. Según Vattimo, lejos de un trascendentalismo romántico, la angustia en el arte surge como "ejercicio de mortalidad" (152) por el que el hombre experimenta sus límites dentro de una sociedad homogeneizada.

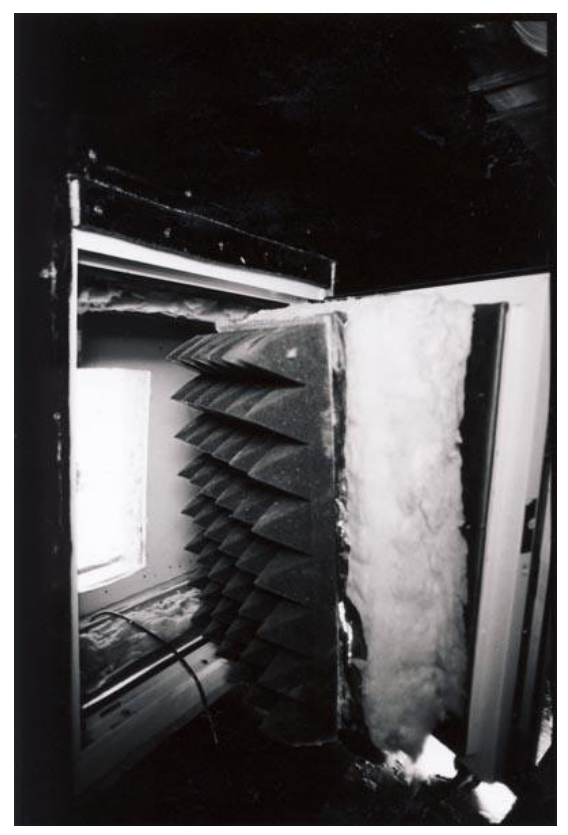

Gregor Schneider. Keller, Haus u r. Rheydt 1992, (c) Gregor Schneider / VG Bild- 
En estos lineamientos, pareciera ser posible responder a la interrogante principal de este trabajo: ¿Es posible repensar la hibridez del espacio estético contemporáneo teniendo en cuenta su devenir e inasibilidad formal? La respuesta es afirmativa mediante la relación arte-angustia, que en los deseos de Bacherlard constituye la posibilidad de referirse al espacio de manera anterior al logos. A diferencia del espacio habitual, el cual emerge del trato del Dasein con el ente a la mano, el espacio estético no se encuentra en las operaciones que implican la desestructuración o intervención de una casa, sino en la posibilidad de ser su propia referencia. Esto, debido a que la verdad que devela la obra quiebra el sistema de significados y representaciones, porque no es universal, sino particular y única. En efecto, la experiencia de recorrer Haus u r produce la vuelta sobre sí del sujeto mediante la retirada de lo cotidiano, en la que está referido a la impropiedad de la ocupación y el uso, efectuando el desplazamiento al espacio existencial, sin referentes ni determinaciones, en el cual la subjetividad queda remitida a la posibilidad de su construcción.

\author{
Universidad Diego Portales* \\ Manuel Rodríguez Sur 415, Santiago (Chile) \\ mpiacordero@gmail.com
}

\title{
OBRAS CITADAS
}

Bachelard, Gastón. La poética del espacio. México: FCE, 2012.

Bourriaud, Nicolás. Estética relacional. Buenos Aires: Adriana Hidalgo Editora, 2006.

Derrida, Jacques. Khora. Buenos Aires: Amorrortu, 2011.

Frampton, Keneth. "Llamado al orden. En defensa de la tectónica". Architectural Design 60, No 3-4 (1990).

Heidegger, Martin. El Origen de la obra de arte. México: FCE, 2006.

— Nietzsche. Barcelona: Destino, 2000.

— Ser y tiempo. Chile: Editorial Universitaria, 1997.

— Kant y el problema de la metafisica. México: Fondo de Cultura Económica, 1996.

— Construir, habitar y pensar [En conferencias y artículos]. Barcelona: Ediciones del Serbal, 1994.

Maldiney, Henry. "Originariedad de la obra de arte". Brumaria, prácticas artísticas, estéticas y políticas. Documento 273. Fecha de ingreso: 6 de agosto, 2014. http://www.brumaria.net/wp-content/uploads/2012/08/273-Maldiney.pdf.

Vattimo, Gianni. La sociedad transparente. Barcelona: Paidós, 1998. 